\title{
Theory Summary and Future Directions
}

\author{
David J. Gross * \\ Physics Department \\ Princeton University \\ Princeton, New Jersey, 08544
}

\section{INTRODUCTION}

Since I am the last speaker, I would like, on behalf of all the participants, speakers and listeners, to thank the organizers of this extremely well organized, well run and stimulating conference at such a beautiful location. I would also like to thank all the other speakers for giving such marvelous talks; especially the theorists, who have given wonderful summaries of the state of particle theory, leaving me to summarize the summaries and review the reviews. The only thing that is really left for me to do is to tell the jokes and provide what our ex-president called "the vision thing". Unfortunately, given the clouds that hang over our field due to the short-sightedness of the U.S. House of Representatives, it is difficult to produce either jokes or vision. But I'll try. What I propose to do is to discuss what some of the highlights and lessons of this conference, mostly those that have to do with theoretical developments, problems and prospects.t]

The main message from this meeting, as well as all other meetings in the last few years, is that the standard model - the electroweak theory and QCD — works extremely well. We are now testing it to unprecedented accuracy as well as deepening our understanding of electron-electron, electron-proton, proton-proton, scattering and exploring the spectrum and

\footnotetext{
${ }^{*}$ Supported in part by the National Science Foundation under

Grant PHY90- 21984.

${ }^{1}$ I shall follow the standard practice of refering all references to the preceeding talks.
} 
the decays of hadronic states.

The success of the standard model is good news. You hear often hear people complaining at meetings like this that the standard model works too well. This agreement of the theory with experiment makes many physicists unhappy. This is understandable. Experimentalists want to discover new phenomena and new laws of physics and besides they want to prove that the theorists are wrong. Theorists, of course, want to get clues as to what the new phenomena and the new laws are and besides they want to prove that the other theorists are wrong. But we should stop complaining about the success of the standard model, because after all the purpose of all of our hard work is to discover the phenomena of nature - what goes on, and then to understand the laws of nature- how it works and why it works. We should be proud and happy that we have a theory that is very successful at what we now refer to as low energy physics (less than a Tev) and that it provides a coherent picture of the particles and their interactions that we can understand. That is, after all, what our effort is all about. We should not be worried that there will not be new physics, we know that there will be new physics. The inadequacies of the standard model and many other clues give us indication that there will be new physics at higher energy and most likely not too far away. If our representatives give us the means to look for the new physics then we will find it. The very success and power of the standard model is basic and necessary if we are to succeed in finding the new physics and make fundamental new discoveries. You must know a lot if you hope to discover something profoundly new.

By now, given the enormous success of the standard model, we should come up with a new name for this theory. The "standard model" is not a good name for what is after all a comprehensive theory of low energy physics. The name, standard model, was invented, in the early seventies. I think it was first used by Steve Weinberg to refer to the $S U(2) \times$ $U(1)$ electroweak theory and to differentiate it from its few rivals, based on the $S U(3)$ and $S U(2)_{R} \times S U(2)_{L}$ gauge groups, alternatives that were still viable at that time. Later in the middle seventies, with the emergence of QCD, it became the name for the combination of the electroweak theory and quantum chromodynamics. However, the situation has changed. 
There are no alternatives to either $S U(2)_{R} \times S U(2)_{L}$ or to QCD. The standard model works and is well tested. It is clear by now that it is not a model in any case; it is a full fledged theory, which is being tested, in many cases, with an accuracy of better than a tenth of a percent, with sensitivity to higher loop effects.

We need a new name that captures the dignity of this theory. It is very hard to come up with a name and I have not done very well. I have a few suggestions: Quantum Particle Theory or perhaps QPT (the name must have a good acronym); or QPD, Quantum Particle Dynamics, for the combination of quantum chromodynamics and quantum flavor dynamics. Another suggestion, in honor of one of the missing ingredients of the standard model, the top quark, is TOP — namely the Theory of Particles. Michael Dine has a nice suggestionTONE, for the The Theory of Nearly Everything. These are only tentative suggestions. Perhaps we should have a contest to come up with a replacement for the standard model. Meanwhile, I will continue to use the standard name.

Another joke. This came up during lunch conversations about the gloomy outlook for the SSC. Someone had the idea that we should look for private funds to support the SSC, just as astronomers convince private individuals to finance large telescopes at a level of a hundred million dollars. But how to attract such money? The idea was that in order to finance the SSC we could auction the quarks. We have given the quarks peculiar names up, down, strange, charm, top and bottom. However we could now sell the names of the quarks for large contributions to the SSC. If you had enough money you might be willing to spend a billion dollars to go down in history as a quark. We might raise a few billion dollars that way. The only problem with this idea is that instead of reporting that CLEO has observed the radiative transition of the bottom quark to a strange quark, we would have to say that they've seen the radiative decay of the David Rockefeller quark to the Ross Perot quark. Maybe it is not worth a few billion dollars! 


\section{ELECTROWEAK INTERACTIONS}

The electroweak theory, as has been widely reported at this conference, passes all tests with increasing and impressive accuracy. The accuracy is now often at the level of .1\% to1\%, most importantly they are beginning to be sensitive to higher order radiative corrections. This is quite a remarkable achievement; it is staggering if one compares the situation with what it was twenty, or even ten years ago. One of the examples of the confrontation between experiment and theory is measurement of the weak angle and the $\rho$-parameter:

$$
\begin{aligned}
\sin ^{2}\left(\theta_{\text {lept. }}\right)=.2321 \pm .0006[\mathrm{EXP} .] ; & =.2332 \pm .0013[\mathrm{TH} .] \\
\rho_{\text {lept. }}=1.0035 \pm .0036[\mathrm{EXP} .] ; & =1.0026 \pm .0038[\mathrm{TH} .]
\end{aligned}
$$

Many more examples were given in talks at this meeting.

At this level of comparison with theory, the precision tests of the standard electroweak theory can be used for many purposes. We can use the fact that the measurements agree with the calculated radiative corrections to constrain the existence of particles that we cannot observe directly but can rule out indirectly. One famous example is the limitation on the number of light neutrinos. In addition there are strong limitations on the masses and couplings of extra $U(1)$ gauge bosons (Z's), and on supersymmetric particles. Most amusingly, these tests can be regarded as providing indirect measurements of the top quark mass. The preferred value is $164 \pm 17 \mathrm{Gev}$ and the range is rapidly narrowing. Given that the precision tests are becoming so accurate and the search for the top quark mass is proceeding so slowly, it seems that we will know the mass of the top quark to better than ten percent before we discover it directly. This is quite a remarkable achievement, especially when we note that we have absolutely no theoretical understanding as to the origin of the magnitude of quark masses.

In addition, the precision tests of the electroweak theory are beginning to be important tools in ruling out or confirming various theoretical ideas as to the origin of the electroweak symmetry breaking or about non-standard model physics. It is fair to say that technicolor 
has been killed by these experiments. This is a nice example of a moderately appealing theoretical idea that can be and has been ruled out by experiment. On the other hand, the predictions of the minimal standard model or of the minimal supersymmetric extensions of the standard model are quite consistent with the precision tests. Most important, these precision tests are beginning to provide one with a very solid spring board towards extrapolation, towards unification at very high energies. In particular the famous coupling constant unification at energies near the Planck scale gives comfort to believers in supersymmetry and in string theory.

\section{QUANTUM CHROMODYNAMICS}

We now turn to QCD-the other component of the standard model. We have heard a lot about tests of QCD in this meeting as well as measurements of properties of hadrons of various types. My impression of all of this is that there has been remarkable progress in the last few years in testing QCD in various processes- deep inelastic scattering, jet production, etc. The confrontation of experiment with theory has been rendered more meaningful by high order calculations. I agree completely with Frank Wilczek's conclusion-QCD is right. In addition to testing QCD, there are many new measurements of hadron physics. Most impressive at this meeting was the enormous amount of data on heavy quark spectroscopy and heavy quark decays. The experimental measurements are impressive and so are the theoretical approaches to this subject. The newly developed heavy quark effective theories have proven to be extremely useful in understanding heavy quark spectroscopy. Lattice gauge theory has developed greatly in the last few years, not just because of the great

increase in the power of modern computers, but also because lattice theorists are beginning to ask new and interesting questions and develop more powerful calculational methods. Finally there has been a lot of progress in using QCD as a theoretical tool for background calculations to ongoing experiments at high energies and for planning new experiments at the new accelerators. Other uses of the theory are for nuclear physics and for nuclear matter 
under unusual circumstances; and as a basis of attempts to construct cosmological scenarios, for example the attempt to understand the origin of baryon number.

I want to make one remark about tests of QCD which has to do with a sum rule to which I am particularly attached to. When I first visited CERN in 1969, I collaborated with a young postdoc there- we were all young in those days-who has since gone on to an administrative position at CERN. The (Gross-Llewyllyn-Smith) sum rule, which we derived using free field current commutators, or equivalently using the parton model, was interesting because it provided a direct test of whether the constituents of the proton had baryon number equal to one-third. It states that

$$
\frac{1}{2} \int d x F_{3}^{\nu p+\bar{\nu} p}\left(q^{2}, x\right)=3
$$

At the time Perkins and his group were doing neutrino experiments at CERN and were very interested in checking this sum rule. They concluded from their data that it was correct with an accuracy of about $100 \%$ (a factor of 2). That was good enough for us to believe immediately in its validity and was one of the reasons that convinced me that quarks really existed inside protons. It is very enjoyable to come to a this conference 24 years later and to hear the sum rule is now being tested to an accuracy of $\sim 2 \%$. As Frank noted, the moments of $F_{3}$ yield some of the best tests of the scaling violations predicted by asymptotically free gauge theories, and we have seen that these are now tested to the level of $2 \%$. But the value of the sum rule does not come out to be 3 but rather $2.50 \pm 0.018 \pm 0.078$. This was compared in this conference with the predictions of QCD, which of course gives corrections to our sum rule of order $\alpha_{s}\left(q^{2}\right)$; in one loop the corrections reduce the sum rule to be equal to $2.66 \pm 0.04$. It was remarked that this represented a slight discrepancy between theory and experiment. I would like to to correct this impression. QCD is not just one loop. Remarkably, the calculation of the sum rule has now been carried out to three loop order, with the result that,

$$
\begin{aligned}
\frac{1}{2} \int d x F_{3}^{\nu p+\bar{\nu} p}\left(q^{2}, x\right) & =3\left[1-\frac{\alpha_{s}}{\pi}-\frac{43}{12}\left(\frac{\alpha_{s}}{\pi}\right)^{2}-18.9757\left(\frac{\alpha_{s}}{\pi}\right)^{3}+\ldots\right] \\
& \sim 2.55 \pm 0.05
\end{aligned}
$$


The answer again is in agreement with experiment, with an accuracy of $\sim 2 \%$.

Some of the other outstanding problems withthe comparisons of QCD with experiment, those having to do with the the EMC effect and the validity of the Bjorken sum rule, seem to be on the verge of being resolved.

Let me say a word about the theoretical situation in QCD. All progress that has been made in QCD relies on the existence of small parameters. To paraphrase Maurice Chevalier: thank heaven for small parameters. In fact, most of our understanding and comprehension of physics relies on the existence of small parameters. The most important small parameters in physics, that determine the structure of the macroscopic world are :
a. $\frac{M_{W}}{M_{\text {Planck }}} \sim 10^{-16}$.
b. $\frac{\Lambda}{M_{\text {Planck }}^{4}} \leq 10^{-120}$.
c. $\frac{M_{\gamma}}{M}=0$.
d. $\alpha \sim 10^{-2}$.
e. $\frac{M_{e}}{M_{p}} \sim 10^{-3}$.

The values of these parameters are mostly mysterious. Nonetheless, the existence of these small numbers is essential for our understanding of the physical universe. The small value of ordinary masses to the Planck mass, the "hierarchy problem", is the reason that gravity is such a weak force weak at low energy. The cosmological constant is probably zero, certainly incredibly small-but we know not why. Similarly the small ratio of the electron mass to the proton mass, or the value of the fine structure constant have no explanation. The only one of the small parameters we do understand is the vanishing photon mass, a consequence of an unbroken gauge symmetry.

If it was not for the existence of these small parameters we would have had a lot of difficulty in developing and understanding physics. We would also not be here. For example, because of (a) gravity is very weak and we can ignore it at low energies. This is good because it is a complicated force. Due to the large hierarchy strings look like particles at low energy, so that we have had the luxury of first understanding simpler particle theories before having to face up to understanding string theory. Another consequence of the hierarchy is that big 
stars, planets and macromolecules can exist. If the Planck mass and the proton mass were comparable then stars containing more than a few nucleons would collapse to black holes.

Because of (b), the smallness of the cosmological constant the universe is so big. If $\Lambda$ had its natural size the the universe would be tiny and we would not be here. Because of(c), (d) and (e) the macroscopic objects exist and in the macroscopic world, to a good approximation, classical non-relativistic physics is a good approximation. Much of the development of physics relies on a series of approximations and hierarchies that follow from the existence of these small numbers.

The same thing is true in QCD, a beautiful but complicated theory. All of the progress that we have made, as well the progress that we envisage in understanding QCD is based on the existence of small parameters. What are the small parameters in QCD? After all QCD is a theory that contains no adjustable parameters, except for the masses of the quarks which are not truly important to the dynamics of the theory. The gauge coupling is not a free parameter; it runs with energy. Nonetheless, there do exist many small parameters in QCD, whose existence has helped us considerably.

The first set of small parameters is are the ratio of the light quark masses relative to the QCD mass scale: $m_{u}, m_{d} / \Lambda \sim 1 / 20$. We might even include the strange quark mass in this list. It is because these masses are so small that chiral $S U(3) \times S U(3)$ is an approximate symmetry of the strong interactions. $S U(3) \times S U(3)$ is not a fundamental symmetry of QCD; rather it is an accidental consequence of the small quark masses. Nonetheless it played a crucial historical role in discovering quarks and understanding the structure of the strong interactions leading to QCD. It continues today to provides the basis for an extremely useful tool-chiral perturbation theory.

The second small parameter is the strong coupling constant $\alpha_{s}\left(Q^{2}\right)$ at large momentum transfers, which, due to asymptotic freedom, is small . This small parameter is even more useful than the others since it is adjustable. One can always make it smaller by increasing the energy, although it only decreases logarithmically. The fact that this parameter is $\sim 1 / 10$ at high energies means that we have the ability to do calculations with the aid of ordinary 
perturbation theory. Much evidence of the agreement of these calculations with experiment was presented in this meeting.

Recently it has been understood that there is another small mass parameter, which is very useful to exploit, namely the ratio of the QCD mass scale to a heavy quark mass, such as the charm quark or the bottom quark. This small parameter is of order of $\sim 1 / 5$ to $1 / 15$. When this parameter is small, i.e. when $N_{Q}$ quark masses are much larger than the QCD mass scale, then QCD acquires an extra $S U\left(2 N_{Q}\right)$ symmetry. This is a very beautiful recent development, only three years old, whose applications to the enormous amount of emerging data on heavy quark spectrum and decay amplitudes have recently exploded. The basic observation is that in the limit where the heavy quark has an infinite mass compared to the QCD mass scale, then when one considers properties of hadrons consisting of some number of heavy quarks, one can neglect the momentum of the light quarks compared to the mass of the heavy quarks. This can be summarized by an effective theory, called the heavy quark effective theory, which has an enhanced symmetry- an $S U\left(2 N_{Q}\right)$ symmetry group which rotates the flavor and spin degrees of freedom of the heavy quark. The heavy quark, being so massive, just sits in the hadrons as a static color source; none of the physics depends on its flavor or on its spin-thus the symmetry. We heard in this meeting of the very beautiful and successful applications of this symmetry to discussing, cataloging and analyzing the spectrum of heavy quarks and their leptonic and semi-leptonic decay amplitudes. When combined with chiral symmetry, useful in analysing the decay amplitudes of B mesons to states containing pions which are the Goldstone bosons of the chiral symmetry, you get very powerful tools. This is a marvelously beautiful subject that is developing rapidly. Unfortunately, like most symmetries, it does not tell you much more more about the theory than that it contains this symmetry. Nonetheless, it is something that the theorists who developed this should be very proud of, a marvelous way of confronting the theory with the enormous amount of data that is now coming out.

Another small parameter that has not been fully explored within QCD is the Bjorken scaling variable $x$, or rather $1 / \ln (1 / x)$. When one measures deep inelastic scattering in 
the region of small $\mathrm{x}$, one is probing a region not dissimilar from the region of diffraction scattering, of Regge behavior. This is the region where another small parameter, namely the ratio of the QCD mass scale to the energy together with ratio of the momentum transfer to the energy are small. This region is now opening up, partly due to the new tool provided by Hera. Hera is a marvelous tool for exploring large $Q^{2}$ physics and testing quantum chromodynamics to very high precision. But it is also a marvelous tool for exploring small $x$ physics where new phenomena might appear. It is amusing that it might very well be that the interesting physics that will emerge from Hera will be the small $x$ physics, unlike the first experiments in deep-inelastic scattering, which revolutionized our view of the strong interactions by discovering scaling at large $Q^{2}$.

At the moment Hera has too low a luminosity to accumulate enough events for precision tests of QCD. However, at small $x$, where most of the cross-section is, and for reasonably large values of $Q^{2}$, they have already observed a quite remarkable phenomenon. They have seen a large number of events with a large rapidity gap, a phenomenon which has also been seen in $D_{0}$ and in UA8. Both groups at HERA have reported that of the order of $5 \%$ of the events with small $x$ and large $Q^{2}$ have a large rapidity gap between fragments of the virtual photon and the fragments of the proton. These events look very different than the standard deep inelastic scattering events that we understand in perturbative QCD. Such events look very much like diffraction scattering, indeed the energy dependence is constant. What is perhaps surprising is that these events scale (i.e. show no power falloff in $Q^{2}$.) This phenomenon is sometimes associated with the hard structure of the Pomeron. The Pomeron, responsible for diffraction scattering for fixed $Q^{2}$, is still one of the most mysterious and least understood aspects of strong interaction physics. There have been many theoretical ideas, mostly from the Leningrad school, which lead one to expect such a phenomenon. I regard this as a very interesting development. The new experimental tool provided by HERA should motivate theorists to once again attack the problem of the Pomeron within QCD and perhaps we might finally get an handle on diffraction scattering.

Another small parameter in QCD is simply $1 / N_{c}=1 /$ the number of colors. This is 
perhaps the nicest of all the small parameters because its value is independent of energy and can be used as an expansion parameter for both short and long range dynamics. Since there are three colors the actual expansion parameter is $\left(1 / N_{c}\right)^{2} \sim 1 / 10$. It is well known that in that in the limit where this parameter is regarded as very small QCD has same qualitative features as it does for $N_{c}=3$, but the calculation of these features becomes much simpler. In fact, there are many $1 / N_{c}$ theorems or relations in QCD that work very well. The $1 / N_{c}$ expansion is perhaps the only hope we have that might allow us total analytical control over the theory. Recently I have been trying to construct, in this $1 / N_{c}$ expansion, a string picture of QCD. That one might hope to describe the infra-red confining dynamics of QCD by means of an effective string theory should not be too much of a surprise. This is an old idea; in fact string theory originated from pion nucleon phenomenology. This is not the fundamental string which contains quarks and gluons, rather a string which describes hadronic flux tubes and which exhibits the nearly linear Regge trajectories. Such a picture is both suggested by hadronic phenomenology and by the $1 / N_{c}$ expansion of QCD. The hadronic string theory (which I call $\mathrm{SCD}=$ String Chromodynamics) would be quite different the fundamental string theory, it would not contain gravitons, gauge mesons or dilatons. My strategy to find the formulation of SCD has far has been to work in two dimensions. Two dimensional QCD much simpler than the real world, but it has many of the same features - confinement, an infinite spectrum of hadrons,etc. The idea is to develop the string theory in two dimensions if possible, It turns out that in two dimensions you can indeed rewrite the theory as a string theory. The plan is then to understand two dimensional SCD well enough to continue to four dimensions. These results are very encouraging but this program is just beginning.

There are other small parameters in QCD that arise if you push the theory to extreme conditions. For example the ratio of the QCD mass scale to the temperature or to the baryon density. If you could heat up quark matter, so that this ratio would be very small, then the vacuum will undergo a deconfining phase transition as well as a chiral symmetry restoring phase transition. At very high temperatures the vacuum should be understandable as a quark gluon plasma. A similar transition should take at high baryon density. The precise 
dynamics in regions of extreme conditions is quite complicated and not that well understood. Here there are potential applications here to heavy ion physics and dense nuclear physics.

Finally there is another small parameter that is exploited all the time, namely is the cost/size of modern computers. This is the parameter that lattice calculations depend on. It is time dependent, decreasing steadily.

\section{BEYOND THE STANDARD MODEL}

Let us now go beyond the standard model. I shall discuss very briefly supersymmetry and string theory. Supersymmetry is rapidly coming into vogue. It is a little scary how rapidly many experimentalists have adopted supersymmetry as the standard non-standard theory. Some people think that is a bad development, since it might mean that they are being hoodwinked by the theorists and are closing their eyes to other possibilities that the theorists have not thought of. I think not. Supersymmetry provides us with definite and relatively precise predictions of almost impossible to measure processes. It therefore poses a real challenge to experimentalists when they design new detectors. The alternative, given the constraints of reality, is not to prepare for all possibilities but for none.

Why do the theorists love supersymmetry? There are at least three reasons. First it is a beautiful extension of ordinary Poincaré symmetry that offers the only known explanation why fermionic matter exists. It makes the existence of fermions as much a consequence of a deep symmetry as the existence of gauge bosons. Second, it is an automatic and necessary component of string theory. In string theory supersymmetry does not just explain the existence of photons; it is required for the existence of fermions. If you want to construct a string theory that contains fermions, it has to be supersymmetric. Finally, most importantly from a phenomenological point of view it offers the most plausible solution of the hierarchy problem, the enormous ratio of the GUT scale to the low energy scale. In this attractive scenario the masslessness of the Higgs boson is protected from developing at the unification scale by supersymmetry, whose breaking then drives the electroweak symmetry breaking 
at a much lower energy. Consequently, this mechanism requires that supersymmetry be observable at low energies. The superscale, that determines the mass splittings between particles and their supersymmetric partners must be of order $100 \mathrm{Gev}$.

The exciting possibility therefore exists that the discovery of supersymmetry is just around the corner, at the next generation of hadron colliders. There is already increasing evidence for supersymmetry. First, the high-precision measurements of the standard model parameters can be used to extrapolate standard model couplings to arbitrarily to very high energy. These extrapolations certainly contradict the simple predictions of minimal GUT's without supersymmetry $(S U(5))$. On the other hand they are in beautiful agreement with the simplest predictions of minimal supersymmetric unification. In this supersymmetric $S U(5)$ model, when the couplings are extrapolated by 12, 13 orders of magnitude, they all come together at a scale of $210^{16} \mathrm{Gev}$, very close to the Planck scale with a coupling that is still reasonably weak $\left(g^{2} / 4 \pi \sim 1 / 26\right.$. $)$ The increase in the GUT scale is due to the screening of the strong coupling by the extra supersymmetric matter particles, therefore pushing its unification with the electroweak coupling to higher energy. This agreement is very nice. Of course it cannot be taken as proof or even as very strong evidence for supersymmetry, but if it had not worked, even to the extent that standard $S U(5)$ does not work, it would be regarded as strong evidence against supersymmetry.

This unification of couplings is also nice in the context of string theory, which is automatically supersymmetric and whose energy scale is usually identified with the Planck mass scale. You might think that there is a disparity, since the unification scale would appear to be quite a bit below the Planck mass scale, which is normally quoted to be $10^{19} \mathrm{Gev}$. However, in the case of the heterotic string theory, the relation between the Planck mass scale $M_{\text {Planck }}$ and the string scale, $M_{\text {string }}$, is dependent on the value of the coupling: $M_{\text {string }}=2 / \sqrt{\alpha^{\prime}}=g M_{\text {Planck }} / \sqrt{8 \pi}$. In addition there exist "threshold corrections", slight shifts in the scale in which the couplings are unified because of the effects that come from integrating out other heavy particles of the theory to one loop order before you make a comparison with the effective low energy field theory. The net result is that one finds that 
the string unification scale is $\sim 310^{17} \mathrm{Gev}$, only a factor of 20 from what is predicted by this 13 to 14 orders of magnitude of extrapolation. I regard this agreement as a great success and a great comfort to string theorists.

Another very nice development that makes supersymmetry look better has to do with the top quark. It is not just that the standard model parameters, when more precisely measured, agree with supersymmetric GUT, but also the fact that the top quark is so heavy makes supersymmetry look much better. One reason is that with a heavy top there is a natural mechanism for producing the electroweak scale of $10^{2} \mathrm{Gev}$ from the GUT scale of $10^{16}$ Gev. This is an old argument, which showed that, in a theory with a heavy top quark, the radiative one loop corrections, arising from the top quark loop, to the Higgs mass matrix, $\Delta m_{\text {Higgs }}^{2} \sim-m_{t}^{2} \ln \left(m_{t} / \mu\right)$ (which are negative because of the fermion loop), can become large enough to drive the Higgs potential unstable and produce a Higgs mass at the electroweak scale. This is a very simple, beautiful and natural explanation of the hierarchy, that becomes credible once the top quark mass is as heavy as it seems to be.

Of course, there are problems with supersymmetry. Most important we do not understand how it is broken, certainly not in the context of string theory. Another problem is that supersymmetric models tend to produce dangerous flavor changing processes. At this conference there was reported the detection of the radiative decay of the bottom quark to the strange quark: $b \rightarrow s \gamma$, which is a threat to supersymmetry since supersymmetric models contain a charged Higgs which can contribute to this decay. The situation is that CLEO has placed a limit $\left(<5.410^{-4}\right)$ on the inclusive branching ratio of the $b \rightarrow s \gamma$ decays, and has observed the decay $B \rightarrow K^{*} \gamma$ with a branching ratio of $(4.5 \pm 1.5 \pm .09) 10^{-5}$. This experiment places a limit of the masses of the charged Higgs $\left(m_{H^{-}}>250 \mathrm{Gev}\right)$, which seems too high for standard SUSY models. I have been told that this problem is easy to circumvent, so it is probably not a a worry. It is interesting that this case stands out because supersymmetry, unlike other nonstandard models, has so far managed to squeak through. At a much more fundamental level, it is very unfortunate that supersymmetry did not provide an answer for the vanishing of the cosmological constant, but that is not really an argument 
against it.

So, in my opinion, the most exciting prospect for the SSC-LHC is the discovery of supersymmetry. Somehow we have been selling these machines to the public in the wrong way. The SSC is not only the machine whose purpose is to discover the Higgs boson but also the instrument that could discover supersymmetry. The United States is going to spend a lot of money on exploring the ordinary three dimensions of space, but at the SSC we will discover not just more of ordinary space but new dimensions of space. Of course, the new dimensions will be fermionic but they are just as real as the ordinary bosonic dimensions of space and time. If we can only convey that feeling of exploration to the public, that we are enlarging space and time, maybe we can convince them to support the SSC.

\section{STRING THEORY}

Turning now to string theory I flash my usual list of the achievements of string theory:

- String Theory provides a consistent, logical and rich extension of the conceptual structure of physics.

- String Theory provides a consistent and finite theory of quantum gravity.

- String Theory provides a rich structure which might yield a unique and comprehensive description of the real world.

Let me discuss the second achievement first. The existence of a finite, consistent theory of quantum gravity within string theory is, at the very least, an existence proof that gravity and quantum mechanics are mutually consistent. Many people suspected that this was not the case, so it is important to have such an example. In addition, stringy gravity is a very interesting theoretical tool. It has motivated the construction of many low energy models of (super)gravity with additional fields such as the dilaton and axion. It has also provided some soluble toy models of two dimensional gravity. These provide a very useful theoretical laboratory for discussing interesting and important conceptual issues of quantum gravity, especially those related to the fate of black holes. 
As for phenomenology, string theory provides interesting unification scenarios that are often quite different from those emerging from ordinary field theoretic GUT's. I think the most interesting thing to focus on is not the detailed prediction of numbers, because you can not trust these, but rather those aspects of nonstandard physics that are nonstandard. These include the existence of discrete symmetries, many possibilities of flavor changing processes, new forms of CP violation, the existence of extra matter: Z's and gauge singlets and the possibility of strange solitons.

We also heard about the very amusing development in which string theory turns out to be a useful way of viewing field theory. After all one can always view some field theories as the low energy limit of string theory. Remarkably, when one looks at field theory in this light one finds that the perturbative calculational devices of string theory organize the field theoretic calculations in a very simple way. This has been fruitfully used to develop improved calculational tools for perturbative QCD.

But I want to come back to the revolutionary aspects of string theory, since I am supposed to talk about vision. There have been two major revolutions completed in this century: relativity, special and general, and quantum mechanics. These were associated with two of the three dimensional parameters of physics: $\hbar$, Planck's quantum of action and $c$, the velocity of light. Both involved major conceptual changes in the framework of physics, but reduced to classical non-relativistic physics when $\hbar$ or $1 / c$ could be regarded as small. The final dimensional parameter is Newton's gravitational constant, that sets the fundamental (Planck) scale of length or energy. Some of us believe that string theory is the revolutions associated with this last of the dimensional parameters of nature. In eventually understanding string theory we will have to undergo a discontinuous conceptual change in the way we look at the world similar to that that occurred in the development of relativity and quantum mechanics.

You might regard this, if true, as very bad news. The evolution of quantum mechanics, from Planck, Einstein and Bohr (1898-1913) to the final theory (1926), required an enormous amount of experimental input. It is very hard to imagine, even with the SSC and much luck, an enormous amount of experimental input that will provide us with direct 
information regarding Planck mass physics. However, we should also remember that the other revolution of this century, that having to do with relativity - special and general—did not require an enormous amount of experimental input. In fact, that conceptual revolution occurred after one already had a theory, Maxwell's theory of electromagnetism (1865), which was a relativistic theory. Einstein only had to understand it better (1905). One can argue that string theory lies somewhere in between these two examples. Every historical revolution (as we have witnessed so dramatically in recent years) is different. The same is true in physics. The development of string theory might a mixture of the quantum and relativistic revolutions. We already have a handle on string theory, the perturbative definition and construction of the theory. But we do not yet have the full fledged formulation of the theory at all. The construction of the ultimate formulation of string theory might require a discontinuous conceptual jump, but we might be able to make this jump just as Einstein could develop relativity from electromagnetism without much experimental input. As you know, the Michelson-Morley experiment, although of fundamental importance, did not influence Einstein very much. What did influence him were Maxwell's equations.

There are many indications that such conceptual jumps will be required in the theory. Most likely we will be forced to change our view of the structure of space-time. One indication that I am particularly fond of is the stringy analogue of the Heisenberg microscope for probing the structure of particles, or the structure of space-time itself. At low energies the accuracy of measurements of position is dominated by quantum mechanics, $\Delta x \sim \hbar / p$, but at high energies the accuracy is dominated by the fact that strings themselves are extended objects. At high energies they smear out, and the Heisenberg uncertainty principle is replaced by,

$$
\Delta x \approx \frac{\hbar c}{E}+\frac{G_{\text {Newton }} E}{g^{2} c^{4}}
$$

Thus the accuracy of position measurements at high energy is limited by a stringy smearing term which is not quantum mechanical. Consequently one can not measure distances smaller than (approximately) the Planck length. 
Other indications of the nature of the conceptual revolution hinted at in string theory abound. One is duality. If strings are compactified on a circle of radius $R$ or on a circle of radius $l_{P}^{2} / R$ one finds identical physics, again suggesting that distances smaller than $l_{P}$ make no sense. This symmetry is very stringy, relating ordinary excitations of the string to solitonic states arising from the winding of the string about the compactified dimensions. Another indication arises from these of the very beautiful mirror symmetry, discussed by Brian Greene, a very stringy symmetry which equates the physical content of apparently different compactified spaces. This symmetry has been used for very mundane things, such as calculating Yukawa couplings and determining fermion masses in superstring models. It has also been used to demonstrate the string physics goes smoothly through a change of topology of space. This is is almost a proof that there is no way one can understand string theory in its full structure within the standard conceptual framework we have of space and time. Another indication arises from the size of nonperturbative effects in string dynamics. There is a connection between the high order behavior of perturbation theory and the nature of the nonperturbative dynamics. In string theory we unfortunately know nothing about nonperturbative dynamics. That is the main reason we can not compare the theory with experiment. But we can construct the perturbation theory. One finds a big difference between the large order behavior of field theory and of string theory. String perturbation theory is of twice as divergent as field theory, which translates into the fact that the nonperturbative phenomena associated with these divergencies of perturbation theory must be different in nature. The magnitude of the non-perturbative effects will be much stronger for small coupling $g, \exp (-1 / g)$ as opposed to $\exp \left(-1 / g^{2}\right)$. Such effects cannot be described by ordinary field theory, non-perturbative string theory will be very different from ordinary field theory. 


\section{PREDICTIONS}

I shall end this talk with a set of predictions. Since this conference is thirty years old I thought I might predict thirty years into the future-but that is much too far. So I will predict halfway, say fifteen years into the future, to the year 2008. First, ten experimental predictions, in order of likelihood:

1. The top quark will be old news and will have a mass of $160 \pm 20 \mathrm{Gev}$.

2. $\epsilon^{\prime} / \epsilon$ will finally be measured and will not be zero. In addition the three B-factories will have found new manifestations of $\mathrm{CP}$ violations.

3. At least 2 light Higgs particles will be found after a few years of running at the SSC.

4. There will be convincing evidence for the existence of supersymmetric particles.

5. The astrophysicists will finally have determined that $\Omega=1$ with an accuracy of $10 \%$. Particle physicists will understand that this mass density is composed of a combination of baryons, axions, neutrinos and neutralinos with various weights (some of which could be zero.)

6. The observation of neutrino oscillations will verify the MSW mechanism and be consistent with the solar neutrino problem.

7. There will be evidence of the quark gluon plasma and of a chiral phase transitions in heavy ion collisions.

8. Some number of new Z-mesons will be discovered.

9. There will be cloudy evidence of superstrings.

10. Finally, the most surprising and strange of all predictions - there will be a real surprise!

Ten more predictions for theory:

1. Lattice gauge theory, armed with Mega-Tera-flop computers, will calculate the hadronic spectrum from QCD with $1 \%$ precision.

2. Analytic treatments in QCD will developed to describe small $x$ physics, Regge behavior and hadronic fragmentation functions. 
3. A nonperturbative treatment of QCD based on the $1 / N_{c}$ expansion and/or stringy QCD will be developed.

4. There will exist a quantitative understanding of the cosmological origin of baryons.

5. There will exist a quantitative understanding of the cosmological origin of density fluctuations leading to the large scale structure of the universe.

6. String field theory will begin to be a useful tool and will illuminate the underlying symmetries of the theory.

7. New mechanisms of string supersymmetry breaking will be discovered leading to new and definitive low energy models.

8. The conceptual revolution arising from the nonperturbative formulation of string theory will be in full swing, revolutionizing the concepts of space-time geometry.

9. The fate of evaporating black holes will be understood without modifing the basic principles of quantum mechanics.

10. And, most unlikely, we will understand why the cosmological constant is zero.

\section{CONCLUSIONS}

I often conclude talks like this one with a discussion of whether we should be optimistic or pessimistic regarding the development of our field. I note that physicists tend to oscillate between extreme optimism and extreme pessimism. They are optimistic when they have discovered new phenomena or a new theoretical technique and are sure that all problems will be cleared up in short order. They are pessimistic when they discover problems with the background or problems with the theory and conclude that these problems are insuperable. They are very rarely moderate. I usually describe myself as being cautiously optimistic,

although I often point out that the optimist is one who proclaims that we have the best of all possible theories and the pessimist is one who fears that this is true.

However, there is a large cloud hanging over this conference, blocking out all the rays of optimism. I will conclude, therefore, with a remark about the SSC. I've been serving on 
the PAC committee at the SSC for the last few years and I have had the opportunity to observe the heroic efforts of our colleagues who are trying to construct a new laboratory and to mount new experiments at this facility. We must do everything possible to help them in these very difficult times. But more than that, we must do everything possible to help ourselves. If the SSC fails, as it might, there would be a tidal that could sweep away our

field. It is very hard to think of strategies for the long term survival of particle physics as an experimental or theoretical field without the SSC.

Since I do not want to end on such a pessimistic note, I will end with David Hilbert's epitaph:

\section{We Must Know- We Shall Know}




\section{QUESTIONS}

Q: I just want to try to understand the modification of the uncertainty principle in string physics. Do you expect this to be changed with the new nonperturbative formulation that you are predicting, or is it pretty much a fixed prediction at the current state of the art?

A: The argument I gave involved much hand waving, much as Heisenberg's arguments were hand waving. Now, within quantum mechanics you can formulate quite precisely what you mean by the uncertainty principle. What I actually believe is that in the new string theory that will eventually arise, much like quantum mechanics replacing the Bohr theory, space and time will not be primary constants. Space and time will emerge as concepts one

can use you use to describe physics under certain circumstances. They will not be primary concepts. But I have absolutely no idea what a precise formulation of the deviations from ordinary geometry will be provided by string physics.

Q: I'd like to ask if there is any prospect of raising money from Congress by selling space on the compactified dimensions?

A: Perhaps. Another suggestion would be to put some of these congressmen, and I have a little list, into those compactified dimensions. 